\title{
Hyperthermic intraperitoneal chemotherapy (HIPEC) and neoadjuvant chemotherapy as prophylaxis of peritoneal carcinosis from advanced gastric cancer-effects on overall and disease free survival
}

\author{
Federico Coccolini ${ }^{1}$, Andrea Celotti ${ }^{1}$, Marco Ceresoli ${ }^{1}$, Giulia Montori ${ }^{1}$, Michele Marini ${ }^{1}$, Fausto Catena $^{2}$, \\ Luca Ansaloni ${ }^{1}$ \\ ${ }^{1}$ General Surgery Department, Papa Giovanni XXIII Hospital, Bergamo, Italy; ${ }^{2}$ General and Emergency surgery, Parma Maggiore Hospital, Parma, Italy \\ Contributions: (I) Conception and design: F Coccolini, A Celotti, L Ansaloni; (II) Administrative support: None; (III) Provision of study materials or \\ patients: None; (IV) Collection and assembly of data: A Celotti, M Marini, G Montori; (V) Data analysis and interpretation: F Coccolini, M Ceresoli, \\ F Catena, L Ansaloni; (VI) Manuscript writing: All authors; (VII) Final approval of manuscript: All authors. \\ Correspondence to: Federico Coccolini, MD. General Surgery Department, Papa Giovanni XXIII Hospital, Piazza OMS 1, 24127, Bergamo, Italy. \\ Email: federico.coccolini@gmail.com.
}

\begin{abstract}
Background: The possibility to enlarge criteria for intra-peritoneal chemotherapy (IPC) to all patients at high-risk to develop peritoneal carcinosis (i.e., with serosal invasion) is still discussed.

Methods: Retrospective case-control study. Three-groups: advanced-gastric-cancer (AGC) (pT4) without proven carcinosis: prophylactic group (PG), those with PC: treatment group (TG), AGC (pT3-pT4) operated without hyperthermic intraperitoneal chemotherapy (HIPEC), surgery alone group (SG T3, SG T4).

Results: Forty four patients. $26(59.1 \%)$ were male. Sixteen (36\%) patients underwent 16 HIPEC: 6 (38\%) had AGC (pT4) without PC (PG), 10 (62\%) had carcinosis (TG), 28 were operated without HIPEC (SG T3, SG T4). The mean disease free survival (DFS): TG: 7.7 months, SG T4: 21.6 months, SG T3: 27.7 months, PG: 34.5 months. DFS was significantly different for TG $(\mathrm{P}=0.03, \mathrm{P}=0.021, \mathrm{P}=0.013$ respectively). The mean OS TG: 10 months, SG T4: 27.1 months, SG T3: 28.2 months, PG: 34.6 months. OS was significantly different for TG (P=0.04, P=0.04, P=0.045 respectively). Severe complication rate: TG: 60\%, PG: 16.7\%, SG T3: 7.7\% and SG T4: 25\% ( $\mathrm{P}=0.035)$. Length-of-stay differs significantly ( $\mathrm{P}=0.003)$; overall length-of-stay: 19.41 days [standard deviation $(\mathrm{SD}) \pm 15.03$ ]; $\mathrm{TG}$ : $33.01(\mathrm{SD} \pm 23.08), \mathrm{PG}: 20.17(\mathrm{SD} \pm 6.21), \mathrm{SG} T 3$ : $11.33(\mathrm{SD} \pm 3.22)$, SG T4: $15.36(\mathrm{SD} \pm 5.48)$.

Conclusions: Prophylactic intraperitoneal chemotherapy associated to neoadjuvant chemotherapy increases the DFS and OS in patients with AGC without carcinosis. More data are needed in order to confirm these results.
\end{abstract}

Keywords: Gastric cancer; intraperitoneal chemotherapy; hyperthermic intraperitoneal chemotherapy (HIPEC); carcinosis; prophylaxis; treatment; prevention

Submitted Mar 29, 2016. Accepted for publication May 16, 2016.

doi: 10.21037/jgo.2016.06.05

View this article at: http://dx.doi.org/10.21037/jgo.2016.06.05

\section{Introduction}

Advanced gastric cancer (AGC) with peritoneal carcinomatosis (PC) generally leads to poor prognosis. The median overall survival (OS) in AGC patients with PC is $9-10$ months. Literature reported no survival at 5 years after resection of the primary tumor $(1,2)$. The $40 \%$ of patients died for GC have hepatic metastases, while the $53-60 \%$ showed a disease progression and died with PC. The two most important factors affecting prognosis in AGC are the serosal invasion and the lymphatic spread (3-5). When gastric serosa is infiltrated, PC could be considered practically unavoidable (6). As a consequence, up to half of 
the patients with AGC will develop PC in spite even radical surgery (7-10). As a counterpart PC is already present in $5-20 \%$ of patients explored for potentially curative resection also in early gastric cancer $(6,11)$. In the Japanese General Rules of Gastric Cancer Treatment, PC are separated into two categories: $\mathrm{P} 0 / \mathrm{Cy} 1$ and $\mathrm{P} 1$ (12). $\mathrm{P} 0 / \mathrm{Cy} 1$ indicates positive peritoneal wash cytology, but no macroscopic PC and P1 indicates the macroscopic PC with or without positive peritoneal cytology. The survival of patients with $\mathrm{P} 0 / \mathrm{Cy} 1$ is similar to that of patients with $\mathrm{P} 1$ (13) and patients with $\mathrm{P} 0 / \mathrm{Cy} 1$ have stage IV disease. This clearly demonstrated as serosal invasion with free cancer cells are comparable in terms of OS to PC. Recent data form a meta-analysis of randomized controlled trials (RCT) demonstrated the benefit to introduce intra-peritoneal chemotherapy (IPC) as preventive or prophylactic treatment of AGC without PC (14). Moreover the necessity to reach a complete cytoreduction in order to increase the survival has been demonstrated by another recent meta-analysis (15).

The possibility to enlarge criteria for IPC also to all those patients at high risk to develop PC (i.e., with serosal invasion) is still discussed even if it has a strong rationale. The aim of the present study is to show the different survival rate between prophylaxis and treatment IPC for PC in AGC and to compare them with the survival of patients with AGC underwent to gastrectomy alone. Several IPC regimens have been applied in the treatment of AGC with PC. No previous reports about the combined use of cisplatin and paclitaxel in hyperthermic regimen exist in AGC. The combination of cisplatin and paclitaxel has been previously described in the management of ovarian cancer $(16,17)$ with good data about its penetration in hyperthermic administration (18).

\section{Methods}

This is a retrospective case-control study, among the 100 patients undergone 100 cytoreduction + hyperthermic intraperitoneal chemotherapy (HIPEC) procedures in our institution from December 2010 to June 2015. Patients with AGC who underwent HIPEC were selected for the present study. Among them, those who had AGC (pT3, pT4) without proven PC constituted the prophylactic group (all pT4) (PG), whereas the remaining ones with proven macroscopic PC constituted the treatment group (TG) (Table 1). A control group of patients with AGC (pT3-pT4) operated in our centre in the same period without being undergone to HIPEC constituted the surgery alone group (SG T3 and SG T4). All patients from the three groups underwent neoadjuvant chemotherapy, gastric resection with or without (respectively depending on the presence or absence of PC) CC-0 cytoreduction and D2 lymphadenectomy. Patients undergone to HIPEC procedures were treated with cysplatin $\left(100 \mathrm{mg} / \mathrm{m}^{2}\right)$ and paclitaxel $\left(75 \mathrm{mg} / \mathrm{m}^{2}\right)$ at $40-41{ }^{\circ} \mathrm{C}$ for 90 minutes with open technique at the end of cytoreductive phase before the reconstructive one. For all the three groups demographic, preoperative, intraoperative, postoperative and histology and follow-up data were recorded. Informed consent has been obtained from each patient and procedures followed were in accordance with the ethical standards of the responsible committee on human experimentation (Institutional or regional) and with the Helsinki Declaration of 1975 , as revised in 1983 .

\section{Statistical analysis}

Data have been prospectively collected in a dedicated database. Continuous variables were expressed as mean, median and [standard deviation (SD)] and were compared with the ANOVA method. Categorical data were expressed as proportion and percentage, and were compared with the Pearson's chi square test. Survivals were calculated with the Kaplan Meier method and differences among subgroups were assessed with the Paired Log Rank Test; $\mathrm{P}<0.05$ was considered statistically significant. Statistics were calculated with SPSS 20 (IBM SPSS ${ }^{\circledR}$ 20, 2012).

\section{Results}

A total of 44 patients were included in the study. Twenty six $(59.1 \%)$ were male. Sixteen (36\%) patients with AGC underwent 16 HIPEC. Among them, 6 (38\%) had AGC (pT3, pT4) without PC (PG), whereas the remaining 10 patients (62\%) had macroscopic PC (TG) (Table 1). Other 28 patients with AGC (pT3-pT4) have been operated without being undergone to HIPEC (SG T3, SG T4).

The overall mean and median age were 65.18 and 70.86 years ( $\mathrm{SD} \pm 14.80)$, in the TG the mean and median age were 50.02 and 48.67 years $(\mathrm{SD} \pm 12.92)$, in the SG T3 73.38 and 74.74 years $(\mathrm{SD} \pm 12.53)$, in the SG T4 70.41 and 70.06 $(\mathrm{SD} \pm 10.29)$ and 59.1 and 60.15 years $(\mathrm{SD} \pm 11.41)$ in the $\mathrm{PG}$.

The mean disease free survival (DFS) time for the TG is 7.7 months, in SG T4 21.6 months, in SG T3 27.7 months and in $\mathrm{PG}$ is 34.5 months. DFS was significantly different only for TG group $(\mathrm{P}=0.03, \mathrm{P}=0.021, \mathrm{P}=0.013$ respectively) (Figure 1). 
Table 1 Included patients data

\begin{tabular}{|c|c|c|c|c|c|}
\hline Variables & HIPEC & Prophylactic HIPEC & Surgery T3 & Surgery T4 & $\mathrm{P}$ \\
\hline $\mathrm{N}$ & 10 & 6 & 14 & 14 & - \\
\hline Age & 48.67 (39.79-60.19) & $60.15(54.70-67.23)$ & $74.74(73.50-76.83)$ & $70.06(63.28-71.75)$ & $<0.0001$ \\
\hline \multicolumn{6}{|l|}{$\mathrm{PCl}$} \\
\hline Mean & $8.9(\mathrm{SD} \pm 9.02)$ & - & - & - & - \\
\hline Hystotype (\%) & & & & & 0.035 \\
\hline Diffuse & 70 & 50 & 29 & 43 & \\
\hline Intestinal & 30 & 50 & 71 & 57 & \\
\hline Complications (\%) & & & & & 0.137 \\
\hline 0 & 20 & 17 & 54 & 58 & \\
\hline 3 & 30 & 0 & 8 & 25 & \\
\hline 4 & 10 & 17 & 0 & 0 & \\
\hline 5 (death) & 20 & 0 & 0 & 0 & \\
\hline Severe complications (3-4-5 CTCAE) (\%) & 60 & 17 & 8 & 25 & 0.035 \\
\hline Overall survival (mean, months) & 10 & 34.6 & 28.2 & 27.1 & - \\
\hline Disease free survival (mean, months) & 7.7 & 34.5 & 27.7 & 21.6 & - \\
\hline Mean length of stay (days) & $33.10 \pm 23.89$ & $20.17 \pm 6.21$ & $11.33 \pm 3.22$ & $15.36 \pm 5.48$ & 0.003 \\
\hline
\end{tabular}

The mean OS time for the TG is 10 months, in SG T4 27.1 months, in SG T3 28.2 months and in PG is 34.6 months. OS was significantly different only for TG group $(\mathrm{P}=0.04, \mathrm{P}=0.04, \mathrm{P}=0.045$ respectively) (Figure 2$)$.

Severe complication rate (Grade 3-4-5 according to CTCAE v4.0) differs significantly between the four different groups $(\mathrm{P}=0.035)$. TG has a complication rate of $60 \%$, PG $16.7 \%$, SG T3 $7.7 \%$ and SG T4 25\%.

Hospital length of stay differs significantly $(\mathrm{P}=0.003)$ between groups. The overall length of stay was 19.41 days ( $\mathrm{SD} \pm 15.03$ ), TG experienced a mean length of stay of 33.01 days $(\mathrm{SD} \pm 23.08), \mathrm{PG}$ of 20.17 days $(\mathrm{SD} \pm 6.21$ ), SG T3 of 11.33 days (SD \pm 3.22$)$ and SG T4 of 15.36 days $(\mathrm{SD} \pm 5.48)$.

\section{Discussion}

Peritoneal carcinosis has been for long time considered as incurable. The last 20 years assisted to the born of the concept of multimodal treatment. It combines systemic chemotherapy, radical surgery and IPC. More recently, the
Peritoneal Surface Oncology Group International (PSOGI) proposed a more complete comprehensive treatment consisting of CRS and perioperative chemotherapy (POC) for the treatment of PC from AGC with curative intent. In PSOGI strategy, peritoneal cancer index (PCI) is determined by laparoscopy, and a peritoneal port is placed. Starting from 2 weeks after laparoscopy, neoadjuvant bidirectional intraperitoneal/systemic chemotherapy (BISIC) is initiated. After three cycles of BISIC, laparotomy and then peritonectomy combined with hyperthermic IPC are performed to remove all macroscopically observable disease. After all, the patients follow the usual adjuvant chemotherapy protocol. The principal aim of the multimodal treatment in AGC is to prevent and/or to treat efficiently PC. Some cohort studies demonstrated its efficacy in advanced AGC with or without PC (14). Many randomized controlled trials (RCT) confirmed the efficacy of IPC in treating AGC. A few of the same RCTs showed as the IPC has a positive effect in the PC prophylaxis and in reducing the local recurrence rate (8-11,19-35). The PSOGI proposed comprehensive 


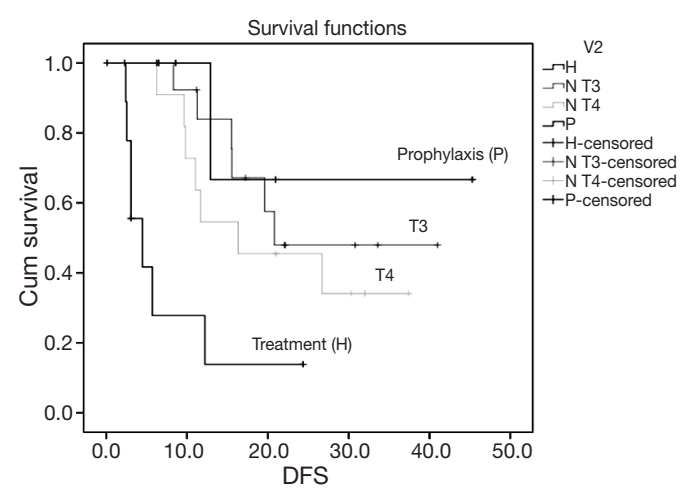

Figure 1 Disease free survival (DFS).

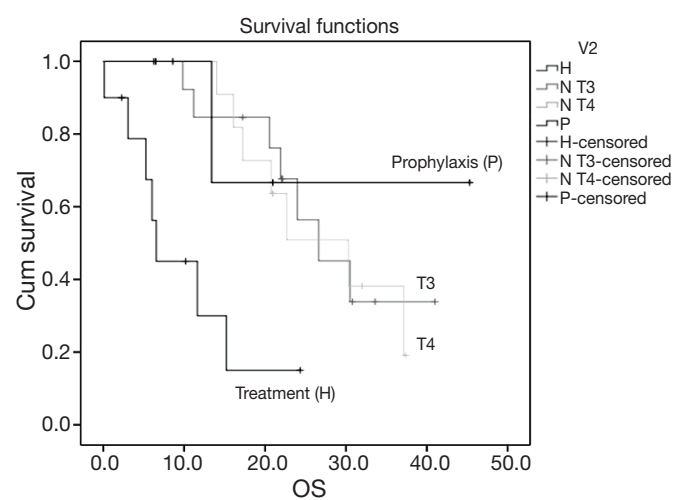

Figure 2 Overall survival (OS).

treatment modality however, even of proven efficacy, results at present not completely accepted by many operators. In this field in fact the majority still looks diffidently to this combination of systems and times to deliver chemotherapy and more in general to the intraperitoneal administration of chemotherapy. This diffidence surprisingly exists despite of AGC is the only disease in which exist level 1 evidences of the effectiveness of IPC (14).

Few previous studies reported promising results about the use of IPC as prophylaxis and treatment of PC by comparing groups of patients affected by AGC with and without PC. A previous analysis has been done about the comparison between these two groups and the major cohort of patients affected by AGC without PC treated only with resection without IPC. This in our opinion represents the most important and significant comparing group (36). In fact the issue consists in introducing the IPC as a prophylaxis of $\mathrm{PC}$ in those patients that are at high risk of developing PC. More in detail, in those patients, the PC is practically unavoidable due to the serosal invasion. The two previous studies that published similar data about the comparison between the treatment and the prophylaxis with IPC of the PC in AGC have been published by Scaringi and by Kang $(36,37)$. Scaringi et al. reported 11 patients with no $\mathrm{PC}$ and 26 patients with macroscopic $\mathrm{PC}$ all undergone to CRS + HIPEC. In the PC-group a complete curative CRS was achieved before HIPEC in 8 patients and a palliative CRS + HIPEC was performed in 18 patients. The median survival was 23.4 months in the no-PC-group, and 6.6 months in the PC-group $(\mathrm{P}<0.05)$. The median survival in the PC-curative subgroup was 15 vs. 3.9 months in the PC-palliative subgroup $(\mathrm{P}<0.007)$. The global recurrence rates between the no-PC-group and the $\mathrm{PC}$-curative subgroup at 2 years were $36 \%$ vs. $50 \%$ respectively. The median delay to recurrence was 18.5 vs. 9.7 months respectively. Kang et al. reported about 83 patients without PC who underwent gastrectomy alone and 29 patients who underwent gastrectomy + HIPEC (36). The reported 5-year survival rates were $10.7 \%$ and $43.9 \%$, respectively. The 5 -year mean survival times were 22.66 (range, 17.55-25.78) and 34.81 (range, 24.97-44.66) months $(\mathrm{P}<0.029)$, respectively. The 3 -year disease-free survival rate for PC was $28.87 \%$ in the group that received surgery alone, whereas it was $66.03 \%$ in the group that received HIPEC. Some RCT also exist about the issue showing good results in terms of survival and recurrence rate. None of these studies however applied a combination of neoadjuvant chemotherapy and subsequent IPC. The present series of patients showed the possibility to apply neoadjuvant chemotherapy followed by a combined regimen of cisplatin and paclitaxel in hyperthermic IPC treatment of AGC with no PC with promising results. Even if not statistically significant, present results clearly demonstrate the effect of this combination of treatments. As a counterpart the clinical significance of these results is encouraging. The lack of statistical significance is mainly due to the small sample of patients in TG and PG. The longer survival is obtained either in comparison with patients treated for PC either in comparison with all those patients who are regularly treated without IPC: the patients with AGC with a T3 or $\mathrm{T} 4$ stage. In fact the vast majority of T3-T4 gastric cancer patients are at high risk of developing PC. The future perspective in the treatment of AGC should be to anticipate the possibility to develop PC. The decision to not keep into consideration the peritoneal fluid cytology derived from the evaluation of the not absolute reliability of the free cells detection and the assumption that serosal invasion is to be considered as practically unavoidable future carcinosis (6). 
The combination of neoadjuvant and intraperitoneal chemotherapy on one hand increases the OS and the DFS and on the other hand doesn't increase significantly over the literature-reported percentages the complication rate and the admission time.

Data from the present study open the necessity to investigate more deeply the use of IPC following the neoadjuvant therapy in the treatment of AGC without PC in order to prevent the relapse and to prolong the survival. Moreover, with the present data, considering a DFS of $47 \%$ in TG and $67 \%$ in PG, with a $95 \%$ interval of confidence, aiming to demonstrate an $20 \%$ increase in 2-year DFS, a randomized controlled trial to compare neoadjuvant chemotherapy + gastrectomy + D2 lymphadenectomy + IPC versus gastrectomy $+\mathrm{D} 2$ lymphadenectomy alone in patients affected by AGC without PC, would need a sample size of 75 patients for each arm. With these numbers, a randomized trial appears feasible and under certain aspects mandatory. One last consideration should be about the application of combined IPC regimens. It has been investigated by previous studies (38). The association of cisplatin and paclitaxel in hyperthermic IPC has been previously described in ovarian cancer $(16,17)$. It demonstrated to not increase the morbidity and the two molecules do not impair each other penetration and effects (18). This is the first report of the use of this association in gastric cancer.

\section{Conclusions}

Prophylactic intraperitoneal chemotherapy associated to neoadjuvant chemotherapy increases the DFS and OS in patients with AGC without peritoneal carcinosis. More data are needed in order to confirm these results.

\section{Acknowledgements}

None.

\section{Footnote}

Conflicts of Interest: The authors have no conflicts of interest to declare.

Ethical Statement: Informed consent has been obtained from each patient and procedures followed were in accordance with the ethical standards of the responsible committee (Bergamo Province Ethical Committee, Papa Giovanni XXIII Hospital) on human experimentation (Institutional or regional) and with the Helsinki Declaration of 1975 , as revised in 1983.

\section{References}

1. Sadeghi B, Arvieux C, Glehen O, et al. Peritoneal carcinomatosis from non-gynecologic malignancies: results of the EVOCAPE 1 multicentric prospective study. Cancer 2000;88:358-63.

2. Bozzetti F, Yu W, Baratti D, Kusamura S, et al. Locoregional treatment of peritoneal carcinomatosis from gastric cancer. J Surg Oncol 2008;98:273-6.

3. Nakamura K, Ueyama T, Yao T, et al. Pathology and prognosis of gastric carcinoma. Findings in 10,000 patients who underwent primary gastrectomy. Cancer 1992;70:1030-7.

4. Takahashi T, Hagiwara A, Sawai K, et al. Intensive Intraoperative Local Chemotherapy for Lymph Node and Peritoneal Metastases in Gastric Cancer. Onkologie 1991;14:152-7.

5. Yu CC, Levison DA, Dunn JA, et al. Pathological prognostic factors in the second British Stomach Cancer Group trial of adjuvant therapy in resectable gastric cancer. Br J Cancer 1995;71:1106-10.

6. Ikeguchi M, Oka A, Tsujitani S, et al. Relationship between area of serosal invasion and intraperitoneal free cancer cells in patients with gastric cancer. Anticancer Res 1994;14:2131-4.

7. Gunderson LL, Sosin H. Adenocarcinoma of the stomach: areas of failure in a re-operation series (second or symptomatic look) clinicopathologic correlation and implications for adjuvant therapy. Int J Radiat Oncol Biol Phys 1982;8:1-11.

8. Hamazoe R, Maeta M, Kaibara N. Intraperitoneal thermochemotherapy for prevention of peritoneal recurrence of gastric cancer. Final results of a randomized controlled study. Cancer 1994;73:2048-52.

9. Ikeguchi M, Kondou A, Oka A, et al. Effects of continuous hyperthermic peritoneal perfusion on prognosis of gastric cancer with serosal invasion. Eur J Surg 1995;161:581-6.

10. Koga S, Hamazoe R, Maeta M, et al. Prophylactic therapy for peritoneal recurrence of gastric cancer by continuous hyperthermic peritoneal perfusion with mitomycin C. Cancer 1988;61:232-7.

11. Kuramoto M, Shimada S, Ikeshima S, et al. Extensive intraoperative peritoneal lavage as a standard prophylactic strategy for peritoneal recurrence in patients with gastric carcinoma. Ann Surg 2009;250:242-6. 
12. Japanese Research Society for Gastric Cancer. The General Rules for Gastric Cancer Study. 1st edition. Tokyo, Japan: Kanahara Shuppan, 1995.

13. Bando E, Yonemura Y, Takeshita Y, et al. Intraoperative lavage for cytological examination in 1,297 patients with gastric carcinoma. Am J Surg 1999;178:256-62.

14. Coccolini F, Cotte E, Glehen O, et al. Intraperitoneal chemotherapy in advanced gastric cancer. Meta-analysis of randomized trials. Eur J Surg Oncol 2014;40:12-26.

15. Coccolini F, Catena F, Glehen O, et al. Complete versus incomplete cytoreduction in peritoneal carcinosis from gastric cancer, with consideration to PCI cut-off. Systematic review and meta-analysis. Eur J Surg Oncol 2015;41:911-9.

16. Coccolini F, Campanati L, Catena F, et al. Hyperthermic intraperitoneal chemotherapy with cisplatin and paclitaxel in advanced ovarian cancer: a multicenter prospective observational study. J Gynecol Oncol 2015;26:54-61.

17. Ansaloni L, Agnoletti V, Amadori A, et al. Evaluation of extensive cytoreductive surgery and hyperthermic intraperitoneal chemotherapy (HIPEC) in patients with advanced epithelial ovarian cancer. Int J Gynecol Cancer 2012;22:778-85.

18. Ansaloni L, Coccolini F, Morosi L, et al. Pharmacokinetics of concomitant cisplatin and paclitaxel administered by hyperthermic intraperitoneal chemotherapy to patients with peritoneal carcinomatosis from epithelial ovarian cancer. Br J Cancer 2015;112:306-12.

19. Wagner AD, Unverzagt S, Grothe W, et al. Chemotherapy for advanced gastric cancer. Cochrane Database Syst Rev 2010;(3):CD004064.

20. Hagiwara A, Takahashi T, Kojima O, et al. Prophylaxis with carbon-adsorbed mitomycin against peritoneal recurrence of gastric cancer. Lancet 1992;339:629-31.

21. Sautner T, Hofbauer F, Depisch D, et al. Adjuvant intraperitoneal cisplatin chemotherapy does not improve long-term survival after surgery for advanced gastric cancer. J Clin Oncol 1994;12:970-4.

22. Fujimura T, Yonemura Y, Muraoka K, et al. Continuous hyperthermic peritoneal perfusion for the prevention of peritoneal recurrence of gastric cancer: randomized controlled study. World J Surg 1994;18:150-5.

23. Takahashi T, Hagiwara A, Shimotsuma M, et al. Prophylaxis and treatment of peritoneal carcinomatosis: intraperitoneal chemotherapy with mitomycin $\mathrm{C}$ bound to activated carbon particles. World J Surg 1995;19:565-9.

24. Fujimoto S, Takahashi M, Mutou T, et al. Successful intraperitoneal hyperthermic chemoperfusion for the prevention of postoperative peritoneal recurrence in patients with advanced gastric carcinoma. Cancer 1999;85:529-34.

25. Rosen HR, Jatzko G, Repse S, et al. Adjuvant intraperitoneal chemotherapy with carbon-adsorbed mitomycin in patients with gastric cancer: results of a randomized multicenter trial of the Austrian Working Group for Surgical Oncology. J Clin Oncol 1998;16:2733-8.

26. Yu W, Whang I, Chung HY, et al. Indications for early postoperative intraperitoneal chemotherapy of advanced gastric cancer: results of a prospective randomized trial. World J Surg 2001;25:985-90.

27. Shimoyama S, Shimizu N, Kaminishi M. Type-oriented intraoperative and adjuvant chemotherapy and survival after curative resection of advanced gastric cancer. World J Surg 1999;23:284-91; discussion 291-2.

28. Yonemura Y, de Aretxabala X, Fujimura T, et al. Intraoperative chemohyperthermic peritoneal perfusion as an adjuvant to gastric cancer: final results of a randomized controlled study. Hepatogastroenterology 2001;48:1776-82.

29. Wei G, Fang GE, Bi JW, et al. Efficacy of intraoperative hypotonic peritoneal chemo-hyperthermia combined with early postoperative intraperitoneal chemotherapy on gastric cancer. Ai Zheng 2005;24:478-82.

30. Yang XJ, Huang CQ, Suo T, et al. Cytoreductive surgery and hyperthermic intraperitoneal chemotherapy improves survival of patients with peritoneal carcinomatosis from gastric cancer: final results of a phase III randomized clinical trial. Ann Surg Oncol 2011;18:1575-81.

31. Miyashiro I, Furukawa H, Sasako M, et al. Randomized clinical trial of adjuvant chemotherapy with intraperitoneal and intravenous cisplatin followed by oral fluorouracil (UFT) in serosa-positive gastric cancer versus curative resection alone: final results of the Japan Clinical Oncology Group trial JCOG9206-2. Gastric Cancer 2011;14:212-8.

32. Ding WT, Duan DM, Sun GS, et al. Clinical observation on early intraperitoneal hypothermic perfusion chemotherapy in the treatment of stomach carcinoma after operation. Tumor 2007;27:585-7.

33. Deng HJ, Wei ZG, Zhen L, et al. Clinical application of perioperative continuous hyperthermic peritoneal perfusion chemotherapy for gastric cancer. Nan Fang Yi Ke Da Xue Xue Bao 2009;29:295-7.

34. Zuo Y, Xu M, Shen D, et al. Postoperative intraperitioneal hyperthermic chemoperfusion combined with intravenous chemotherapy for 82 advanced gastric cancer patients. Zhonghua Zhong Liu Za Zhi 2004;26:247-9.

35. Tan CQ, Wang XJ, Xu YZ, et al. Clinical studies on 
prevention of post-operational intraperitoneal neoplasm seeding of advanced gastric cancer by surgery combined with intraperitoneal perfusion of hyperthermic mitomycin C. Journal of Practical Oncology 2000;15:165-8.

36. Kang LY, Mok KT, Liu SI, et al. Intraoperative hyperthermic intraperitoneal chemotherapy as adjuvant chemotherapy for advanced gastric cancer patients with serosal invasion. J Chin Med Assoc 2013;76:425-31.

Cite this article as: Coccolini F, Celotti A, Ceresoli M, Montori G, Marini M, Catena F, Ansaloni L. Hyperthermic intraperitoneal chemotherapy (HIPEC) and neoadjuvant chemotherapy as prophylaxis of peritoneal carcinosis from advanced gastric cancer-effects on overall and disease free survival. J Gastrointest Oncol 2016;7(4):523-529. doi: 10.21037/ jgo.2016.06.05
37. Scaringi S, Kianmanesh R, Sabate JM, et al. Advanced gastric cancer with or without peritoneal carcinomatosis treated with hyperthermic intraperitoneal chemotherapy: a single western center experience. Eur J Surg Oncol 2008;34:1246-52.

38. Braam HJ, Schellens JH, Boot H, et al. Selection of chemotherapy for hyperthermic intraperitoneal use in gastric cancer. Crit Rev Oncol Hematol 2015;95:282-96. 